\title{
Electrically Tunable Delay for Trains of Optical Pulses
}

\author{
Santiago Tainta , María J. Erro' , María J. Garde' , Miguel A. Muriel
}

\begin{abstract}
A technique to implement an electrically tunable delay line with high bandwidth for trains of ultrashort optical pulses is presented. The system is based on the temporal self-imaging effect in fiber gratings and electrooptic modulation.
\end{abstract}

\section{Introduction}

Optical delay lines are key elements in different applications, such as optical signal processing, buffers and synchronization devices in optical communication systems, or Optical Coherence Interferometry (OCT). Current techniques addressing delay lines and their tuning can be grouped in two categories. First, those based on the socalled slow-light effect achieved by using waveguides based on photonic crystals or resonant elements [1], which tune the delay by varying the effective refractive index. A second approach uses dispersive elements whose delay depends on wavelength. Additional techniques for wavelength conversion and to avoid distortion are required in this case to tune the delay [2]. In this work, a new technique is presented for a fiber-integrated delay line which does not use resonant elements and operates on optical pulses trains. It results in a broad bandwidth scheme where elements for wavelength conversion are avoided. The delay is tuned by simply modifying an electric signal that is used to modulate the input pulse train.

\section{Proposed setup}

The proposed system operates on an ultrashort optical pulse train and is shown in Fig. 1. A Mode Locked Laser (MLL) is used to generate a $T_{0}$-repetition rate pulse train. This input train of pulses can be considered as a train of Dirac delta functions evenly distributed in time, as the pulse duration is chosen to be much lower than the period of repetition. This pulse train is then phase-modulated using an Electrooptical Phase Modulator (EO-PM) with halfwave voltage given by $V_{\pi}$. The modulating signal is chosen to be a signal linear in time, whose constant slope we designate as $C$. The modulated train is then input to a dispersive medium, a Linearly Chirped Fiber Bragg Grating (LC-FBG) in our case. Its dispersion, $\ddot{\phi}$, is to be selected so that it results in the temporal self-image effect with $m=1$ and $s=1$ parameters, as defined in [3], which corresponds to $\ddot{\phi}=T_{0}^{2} /(2 \pi)$.

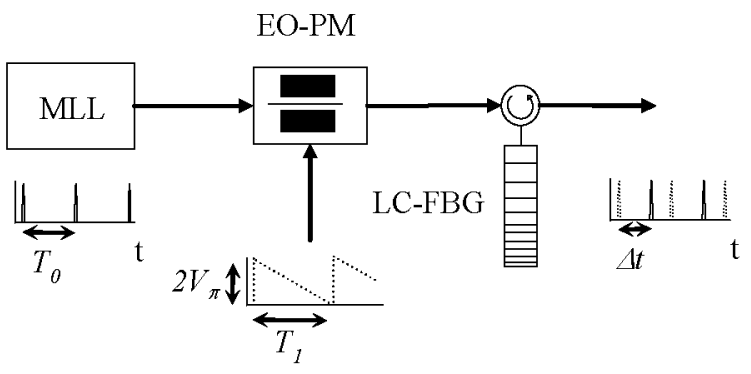

Fig. 1: Proposed setup.

If this dispersion was applied to a non-modulated pulse train, the output would be a pulse train with the same rate but delayed by $T_{0} / 2$ [3]. Here, where a linear phase modulation is induced, the output, $x(t)$, can be shown to be:

$$
x(t) \propto \exp \left(j \frac{t^{2}}{2 \ddot{\phi}}\right) \sum_{k=-\infty}^{\infty} \delta\left(t-\Delta t-k T_{0}\right)
$$

where

$$
\Delta t=\frac{T_{0}}{2}\left(1+\frac{C}{\pi} T_{0}\right)
$$


However, a time linear electric signal can not be generated but for a finite time interval. Hence, this signal is replaced by a ramp with amplitude given by $2 V_{\pi}$ and a repetition rate given by $T_{1}$. This ramp, given the inherent periodicity of phase modulation, is equivalent to a linear modulation with a slope $C= \pm 2 \pi / T_{1}$, where the slope sign depends on the ramp direction. Moreover, in order to avoid the transitions between periods of the ramp so that a linear modulation can be assured, the optical pulses have to be placed only in the linear zones of the ramp. This can be achieved by limiting the ramp period to an integer multiple of the original pulse train period, $T_{1}=k T_{0}$, where $k$ is an integer number. With these conditions, the overall delay at the system output is deduced from eq. (2) to result in

$$
\Delta t=\frac{T_{0}}{2}\left(1 \pm \frac{2}{k}\right)
$$

Eq. (3) represents the sum of a constant term due to the self-image effect and a variable term inversely proportional to $k$, that is, proportional to the frequency of the electric ramp signal. Additionally, both positive and negative delays can be obtained as a function of the slope sign. Delay values, on the contrary side, are limited to a discrete set of values as a function of $k$. This drawback could be avoided with the use of an arbitrary shape wave generator, which could provide a phase bias to every pulse in an equivalent way as an ideal ramp would.

\section{Results}

A set of simulation runs were carried out using Optsim ${ }^{\mathrm{TM}}$ in order to validate the proposed system. As the pulsed source, a MLL with $10 \mathrm{GHz}$-repetition rate and 5 ps-width Gaussian pulses was used. Laser output was modulated with an electrooptical phase modulator featuring a $12.5 \mathrm{GHz}$ bandwidth, $3 \mathrm{~dB}$ insertion losses, and $5 \mathrm{~V}$ half-wave voltage. In consequence, a $10 \mathrm{~V}$ amplitude ramp was applied as modulating signal, and its frequency was then varied from 0.5 to $10 \mathrm{GHz}$ to see the effect on the delay. Both positive and negative ramps were used. The modulated signal is then dispersed using a $12.3 \mathrm{~cm}-$ long LC-FBG, chosen to give a $1590 \mathrm{ps}^{2}$ dispersion -in good agreement with the required dispersion for $T_{0}=1 / 10 \mathrm{GHz}$ - and about $0.8 \mathrm{~nm}$ bandwidth. Its frequency response was theoretically computed using mode coupling theory with an hyperbolic taper with $\alpha=4$ for smoothing.

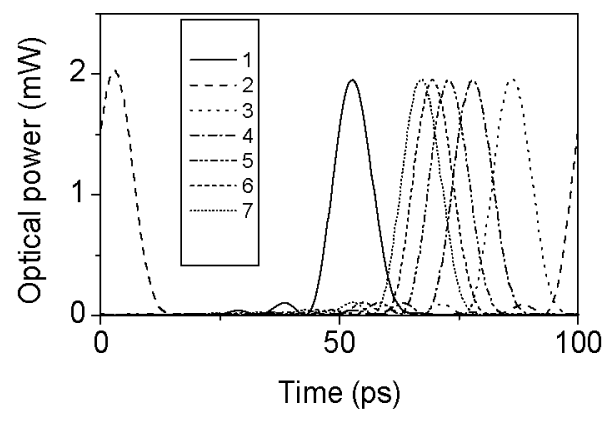

(a)

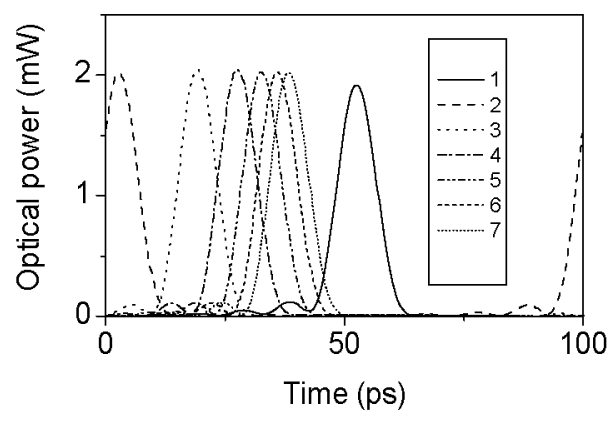

(b)

Fig. 2: Delayed pulse trains at the output for different values of the parameter $k$ for the ramp signal (indicated in the onsets) with (a) positive or (b) negative slope.

Fig. 2 (a) and (b) show the pulses at the system output for different ramp periods. A certain degree of deformation in the pulse shape can be appreciated and it is caused by the filtering introduced by the Bragg grating and the nonidealities in the second order dispersion applied, basically the ripple in the group delay. It can be observed that the resulting delay is inversely proportional to $k$, i.e. directly proportional to the ramp frequency, as predicted by (3), and that positive or negative delays can be obtained depending on the ramp sign. But, being the achievable delay limited to a discrete set of values, the pulse can only be delayed up to half the bit period for each ramp sign. Both slopes are required then in order to tune the pulse over a whole bit period. This effect is shown in Fig. 3, where the delay is represented as a function of the modulating frequency, once the constant term $T_{0} / 2$ is removed. It is clear that both positive and negative ramps with frequencies lower than $5 \mathrm{GHz}$ are required to get a tuning range of the whole bit, that is, $100 \mathrm{ps}$. 


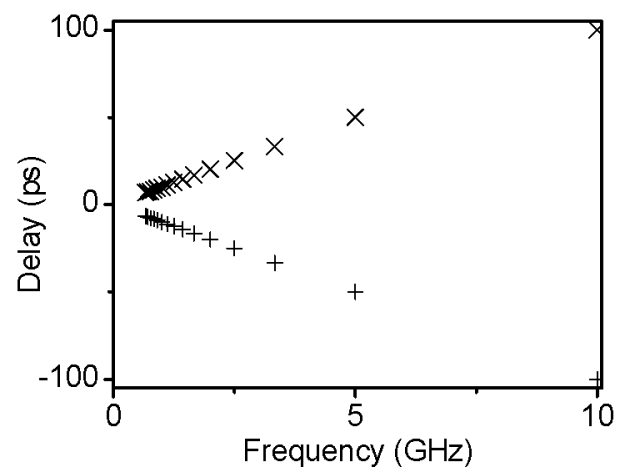

Fig. 3: Different delays introduced for the different values of $k$.

\section{References}

[1] A. Melloni et al, "Tunable delay lines in silicon photonics: coupled resonators and photonic crystals, a comparison," IEEE Photon. J. 2, 181194 (2010).

[2] C. Caucheteur et al, "All-fiber tunable optical delay line," Opt. Express 18, 3093-3100 (2010).

[3] J. Azaña and M.A. Muriel, "Temporal Talbot effect in fíber gratings and its applications," App. Opt. 38, 6700-6704 (1999). 